\title{
Clinical and economic analysis of Gastrodin injection for dizziness or vertigo: a retrospective cohort study based on electronic health records in China
}

Yunfeng Lai ${ }^{1,2+}$, Ruoning Wang ${ }^{3}$, Wei Li ${ }^{4}$, He Zhu ${ }^{4}$, Shuyang Fei ${ }^{5}$, Honghao Shi ${ }^{2}$, Nan Lu ${ }^{6}$, Carolina Oi Lam Ung ${ }^{2}$, $\mathrm{HaO} \mathrm{Hu}{ }^{2^{*}} \mathrm{D}$ and Sheng $\mathrm{Han}^{4^{*}}$

\begin{abstract}
Background: Dizziness and vertigo are common clinical symptoms. Gastrodin injection has shown clinical effects on dizziness or vertigo. However, little is known about the effectiveness and costs of combining Gastrodin injection with conventional treatment on dizziness or vertigo in daily practice. This study aimed to analyze the clinical and economic effects of Gastrodin injection for patients with dizziness or vertigo in comparison to Extract of Ginkgo Biloba Leaves injection in real-world practice.

Methods: Data was collected from the Hospital Information System of 131 hospitals across China from January to December 2018. Patients whose primary discharge diagnosis was dizziness or vertigo according to ICD-10 diagnostic coding were included and divided into two samples: sample of dizziness or vertigo; sample of dizziness or vertigo, with the complication of cerebral infarction. Comparative analysis of the medical cost per hospitalization, hospitalization duration, effective rates, and cure rates between the group of Gastrodin injection and the group of Extract of Ginkgo Biloba Leaves injection was conducted. Propensity Score Matching was used to control potential confounding factors.

Results: In the sample of dizziness or vertigo, although there was no significant differences on hospitalization duration $(P=0.080)$, the group of Gastrodin injection was significantly better than the group of Extract of Ginkgo Biloba Leaves injection $(P<0.001)$ in terms of treatment effect and the per capita hospitalization cost. In the sample of dizziness or vertigo, with the complication of cerebral infarction, there was no significant difference $(P=0.371)$ in terms of hospitalization duration, but the group of Gastrodin injection was significantly better than the group of Extract of Ginkgo Biloba Leaves injection $(P=0.009)$ in terms of treatment effect, and significant difference regarding the per capita hospitalization cost $(P<0.001)$.
\end{abstract}

\footnotetext{
*Correspondence: haohu@um.edu.mo; hansheng@bjmu.edu.cn

†Yunfeng Lai and Ruoning Wang shares first authorship

${ }^{2}$ State Key Laboratory of Quality Research in Chinese Medicine, Institute

of Chinese Medical Sciences, University of Macau, Taipa, Macao SAR,

China

${ }^{4}$ Department of Real-World Evidence and Pharmacoeconomics,

International Research Center for Medicinal Administration, Peking University, Beijing, China

Full list of author information is available at the end of the article
}

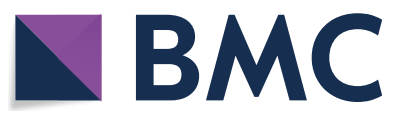
permits use, sharing, adaptation, distribution and reproduction in any medium or format, as long as you give appropriate credit to the original author(s) and the source, provide a link to the Creative Commons licence, and indicate if changes were made. The images or other third party material in this article are included in the article's Creative Commons licence, unless indicated otherwise in a credit line to the material. If material is not included in the article's Creative Commons licence and your intended use is not permitted by statutory regulation or exceeds the permitted use, you will need to obtain permission directly from the copyright holder. To view a copy of this licence, visit http://creativecommons.org/licenses/by/4.0/. The Creative Commons Public Domain Dedication waiver (http://creativeco mmons.org/publicdomain/zero/1.0/) applies to the data made available in this article, unless otherwise stated in a credit line to the data. 
Conclusions: Gastrodin injection showed advantages for inpatients with dizziness or vertigo compared with Extract of Ginkgo Biloba Leaves injection. Future studies using prospective pragmatic controlled trials can test and explore more about the effects of Gastrodin injections on dizziness or vertigo.

Keywords: Gastrodin injection, Dizziness, Vertigo, Extract of Ginkgo Biloba Leaves injection, Electronic health record

\section{Background}

Dizziness is divided into vertigo, presyncope, disequilibrium, and lightheadedness in the world [1-3]. Dizziness and vertigo are the main manifestations of various central or peripheral vestibular syndromes caused by cerebrovascular disease. Dizziness and vertigo are common morbid syndrome, and the prevalence rate increases with age, which are the top three main reasons for the elderly to see a doctor. Patients are often complicated with various complications, rapid development of the disease, and hidden causes, which significantly increased clinical treatment difficulty [4]. According to the anatomic location of the lesions in clinical, vertigo can be divided into systemic vertigo caused by vestibular nervous system lesions and non-systemic vertigo caused by lesions outside the vestibular system [5]. The isolated dizziness or vertigo of cerebrovascular disease is easily misdiagnosed and might cause severe consequences [6]. However, dizziness is the most common clinical symptom in posterior circulation cerebral infarction with the highest incidence [7]. Some studies on the research between incidence rate of acute cerebrovascular disease and isolated dizziness or vertigo have shown that the probability of acute cerebrovascular disease (cerebral infarction or cerebral hemorrhage) with acute dizziness or vertigo, nystagmus and/or balance disorders is $4.6-11 \%[8,9]$.

Specifically, stroke has become the most common high disability and deadly disease globally [10] and the leading cause of death in China in recent years [11]. The age of stroke onset was earlier than before, and the prevalence and hospitalization rate showed a significant upward trend [12]. Cerebral infarction accounts for about $70 \%$ of stroke, and posterior circulation cerebral infarction accounts for about $20 \%$ of ischemic stroke [13]. Therefore, dizziness and vertigo have caused a heavy economic burden on patients and the health care systems worldwide [14].

According to the Multidisciplinary Expert Consensus of Diagnosis and Treatment of Vertigo (2017 edition), routine treatment with western medicine with the anti-inflammatory action of glucocorticoid is the first choice thrombolysis, antiplatelet, and anticoagulation [15]. Gastrodin is a active component extracted from the traditional Chinese medicine Gastrodia elata Blume, called Tianma in Chinese [16]. Gastrodia elata Blume and Gastrodin have low toxicity [17] and have pharmacological activities such as dizziness, stoke, headache, sedative, analgesic and neurological disorder-improving [18-21], which have a potential role in the protection of nerve cells [22,23].

Gastrodin injection is mainly used in the clinical treatment of dizziness, vertigo, stroke, insomnia, headache, neurasthenia, coronary heart disease, and epilepsy [24-26]. The clinical effect is positive, and there is no significant adverse reaction [27]. Compared with western medicine, Gastrodin injection has better effectiveness and safety in treating cerebral infarction [28]. Furthermore, based on a multi-center single-blind randomized controlled trial, Gastrodin injection is safe and effective for vertigo and is better than betahistine injection in relieving vertigo symptoms [29].

Although Gastrodin injection showed sound clinical effects in these studies, there is little known about the clinical and economic evaluations of Gastrodin injection in real-world practice. Some retrospective studies had compared Gastrodin injection with other drugs in the treatment of dizziness and vertigo [30-32]. However, the actual conditions of patients are evidence of clinical medication decisions by doctors. It must consider realistic comparator drugs to evaluate specific medicine products' clinical and economic effects [33]. The leveraging of real-world data can improve clinical evaluation and that is one of the priority areas for improving public safety and facilitating innovation in China [34]. From current literature, there is still lacking studies on the evaluation of curative effect and economic benefit of the Gastrodin injection with the comparison of other drugs for patients in the real clinical environment.

In clinical practice, Extract of Ginkgo Biloba Leaves injection has been widely used for dizziness, vertigo, and cerebral infarction with low toxicity and a good curative effect with little adverse reaction [35-37]. It is a naturally extracted medicine, like Gastrodin injection, which can provide new treatment with high efficiency and low toxicity at low development cost [38]. The development of phytomedicine based on modern medicine and pharmaceutical theory is an innovation and has been recognized [39]. Thus, the comparison between these two naturally extracted medicines is more convincing, and it also can provide practical references for guiding the practice of interchangeability of the same type of drugs. 
Therefore, this study aimed to analyze the clinical and economic effect of Gastrodin injection for patients with dizziness or vertigo in comparison to Extract of Ginkgo Biloba Leaves injection in real-world practice. It's expected that the findings can provide references for clinicians to optimize treatment options.

\section{Methods}

\section{Research design}

This study was a retrospective cohort study to compare the clinical and economic effects of Gastrodin injection and Extract of Ginkgo Biloba Leaves injection for hospitalization patients diagnosed with dizziness or vertigo (see STROBE checklist in Additional file 1).

\section{Composition information}

In 1980, the chemical synthesis of Gastrodin was completed. The State Medicine Administrative Bureau approved Gastrodin injection of China as a new drug and first authorized the KPC Pharmaceuticals, Inc to manufacture in 1984. At present, 25 pharmaceutical companies are producing Gastrodin injection, with 37 approvals (including different specifications).

\section{Botanical}

Gastrodia elata Blume (Orchidaceae) is an obligate mycoheterotrophic plant, an orchid popularly used in traditional Chinese medicine [40]. It is primarily found in China, Nepal, Bhutan, India, Japan, North Korea, Siberia, and Taiwan [41, 42]. In China, it grows mainly in Sichuan, Yunnan, Guizhou, and Hunan Province [43], where can provide high-quality daodi medicinal products [44]. Gastrodia elata Blume is cited by the Pharmacopoeia of China [45] and has been used in oriental medicine in East Asia to treat various diseases, including neurological disorders as an anticonvulsant, analgesic, and sedative medication [46].

\section{Chemical}

Gastrodin injection is a kind of traditional Chinese medicine injection that is extracted and refined from G. elate [28]. The main ingredient is gastrodin and identified by the State Medicine Administrative Bureau of China. Since the early 1950s, China has begun to study the chemical composition of gastrodin. Nearly 100 compounds have been isolated from gastrodin. In 1978, gastrodin was isolated from the ethanolic extract of Rhizoma Gastrodiae for the first time [47, 48]. Through silica gel colum chromatography, 14 gastrodin compounds have been isolated and identified, included 8 phenolic components were fractionated: 4-hydroxybenzaldehyde (4-HBAL), 4-hydroxybenzyl alcohol (4-HBA), benzyl alcohol, bis(4-hydroxyphenyl) methane, 4-(4'-hydroxybenzyloxy) benzyl methylether, 4-hydroxy-3-methoxybenzyl alcohol (vanillyl alcohol), 4-hydroxy-3-methoxybenzaldehyde (vanillin), and 4-hydroxy-3-methoxybenzoic acid (vanillic acid) $[46,49]$.

\section{Target population and data source}

The target population of this study included inpatients whose primary diagnosis of hospital discharge was dizziness or vertigo according to the ICD-10 coding, treated between 1st January 2018 and 31st December 2018. In this study, data was collected from the Health Information System (HIS) of 131 hospitals around China, including 101 tertiary hospitals, 24 secondary hospitals, and 6 primary hospitals.

The specific inclusion criteria of the patients are:

1) Inpatient who diagnosed with dizziness or vertigo between 1st January 2018 and 31st December 2018;

2) Dizziness or vertigo was the principal diagnosis (ICD-10 code: $\mathrm{H} 81$ and R42);

3) Gastrodin injection alone or Extract of Ginkgo Biloba Leaves injection alone in clinical treatment.

The specific exclusion criteria of the patients are:

1) Patients who were younger than 18 years old or older than 75 years old;

2) Patients with cancers or pregnancy;

3) Patient record is incomplete.

Regarding patient inclusion, we selected the data in sequence. First, coding H81 and R42 with ICD-10 was used to filter and diagnose data containing H81 and R42 from the full data set to obtain the Per Protocol Set (PPS). Second, according to the subgroup design, the subgroup data was screened separately. The subgroup of dizziness and vertigo combined with cerebral infarction is a subdata set filtered with the keyword "cerebral infarction" from PPS.

\section{Sampling}

The included patients were divided into two samples: sample of dizziness or vertigo; sample of dizziness or vertigo complicated with cerebral infarction.

- Sample of dizziness or vertigo

Patients were with the only principal diagnosis as dizziness or vertigo.

- Sample of dizziness or vertigo, with the complication of cerebral infarction 
Patients were with the principal diagnosis as dizziness or vertigo, and with another diagnosis as cerebral infarction.

\section{Treatment}

All patients were divided into exposed group and control group. The exposed group was hospitalized with Gastrodin injection, and the control group was treated with Extract of Ginkgo Biloba Leaves injection. The outcomes and costs of Gastrodin injection and Extract of Ginkgo Biloba Leaves injection were compared.

\section{- Gastrodin injection}

During hospitalization, the principal diagnosis was dizziness or vertigo and was prescribed Gastrodin injection, while Extract of Ginkgo Biloba Leaves injection and other Chinese medicine injections of dizziness or vertigo were not prescribed.

- Extract of Ginkgo Biloba Leaves injection During hospitalization, the principal diagnosis was dizziness or vertigo and was prescribed Extract of Ginkgo Biloba Leaves injection, while Gastrodin injection and other Chinese medicine injections of dizziness or vertigo were not prescribed.

Due to the lack of therapeutic dosage on the first page of the collected electronic health records, the therapeutic dosage could not be included in the analysis. The clinical experts we consulted indicated that clinicians usually chose therapeutic dosage according to patient status and disease progression in realistic practice. Thus, in this study, we compared the two groups (Gastrodin injection vs. Extract of Ginkgo Biloba Leaves injection), not the therapeutic dosage.

\section{Outcomes}

This study's outcome indicators included medical cost per hospitalization, hospitalization duration, effective rate, and cure rate, which were suggested by the clinical experts we consulted. Therapeutic effects are divided into death, ineffective, effective (turn for the better, cure), and others. Specifically, effective includes turn for the better and cure of clinical treatment. Cure refers to "the cure of each hospitalization", and the cure rate refers to "the cure rate of each hospitalization". The cure rate is based on the realistic observation and judgment of clinical doctors directly responsible for the patients, which is directly extracted from the original medical records in the HIS of the hospitals.

\section{Costs}

In this study, the costs only considered the direct medical cost in the period of 1st January 2018 to 31st December
2018. Including total hospitalization costs, drug fees, laboratory fees, bed fees, operation fees, nursing fees, inspection fees, treatment fees, and other fees. Among them, the cost of drug includes target drug fee (Gastrodin injection or Extract of Ginkgo Biloba Leaves injection) and other drug fee. Cost data collected from HIS of 131 hospitals over the country. This study focused on medical costs per hospitalization because this data is the most reliable data to reflect treatments' economic effectiveness.

\section{Bias and elimination}

Propensity score matching (PSM) is used to control the confounding factors and reduce the impact of confounding factors in evaluating intervention effects. In the study, logistic regression was used to score the tendency, mixed variables including region, hospital level, hospital department, age, and sex. PSM was achieved by Nearest Neighbor Matching (NNM) in the match it bag of R language, where the matching ratio is set to be $1: 1$.

\section{Statistical analysis}

In this study, descriptive statistics analysis was conducted to describe basic characteristics, comparative analysis of outcome, and cost. Descriptive analysis was conducted as measurement data are described by median and quartile, and enumeration data are described by frequency and rate. The outcome, hospitalization cost and composition, percentage, and $P$-value of the two groups were also compared and analyzed by SPSS statistical software. $P<0.05$ was considered statistically significant.

\section{Results}

In this study, 9251 patients with dizziness or vertigo who met the inclusion and exclusion criteria were selected, including 7113 patients in the exposed group and 2138 patients in the control group. After stratified analysis, there were 2078 patients in the sample of dizziness or vertigo; and 1042 patients in the sample of dizziness or vertigo, with the complication of cerebral infarction. The flowchart of sampling was summarized in Fig. 1.

\section{Sample of dizziness or vertigo}

\section{Sample characteristics at baseline before PSM}

As shown inTable 1, 1529 patients were included in Gastrodin injection treatment, while 528 patients were included in Extract of Ginkgo Biloba Leaves injection treatment. In these two groups, there were significant differences in region, hospital level, and hospital department $(P<0.001)$. 


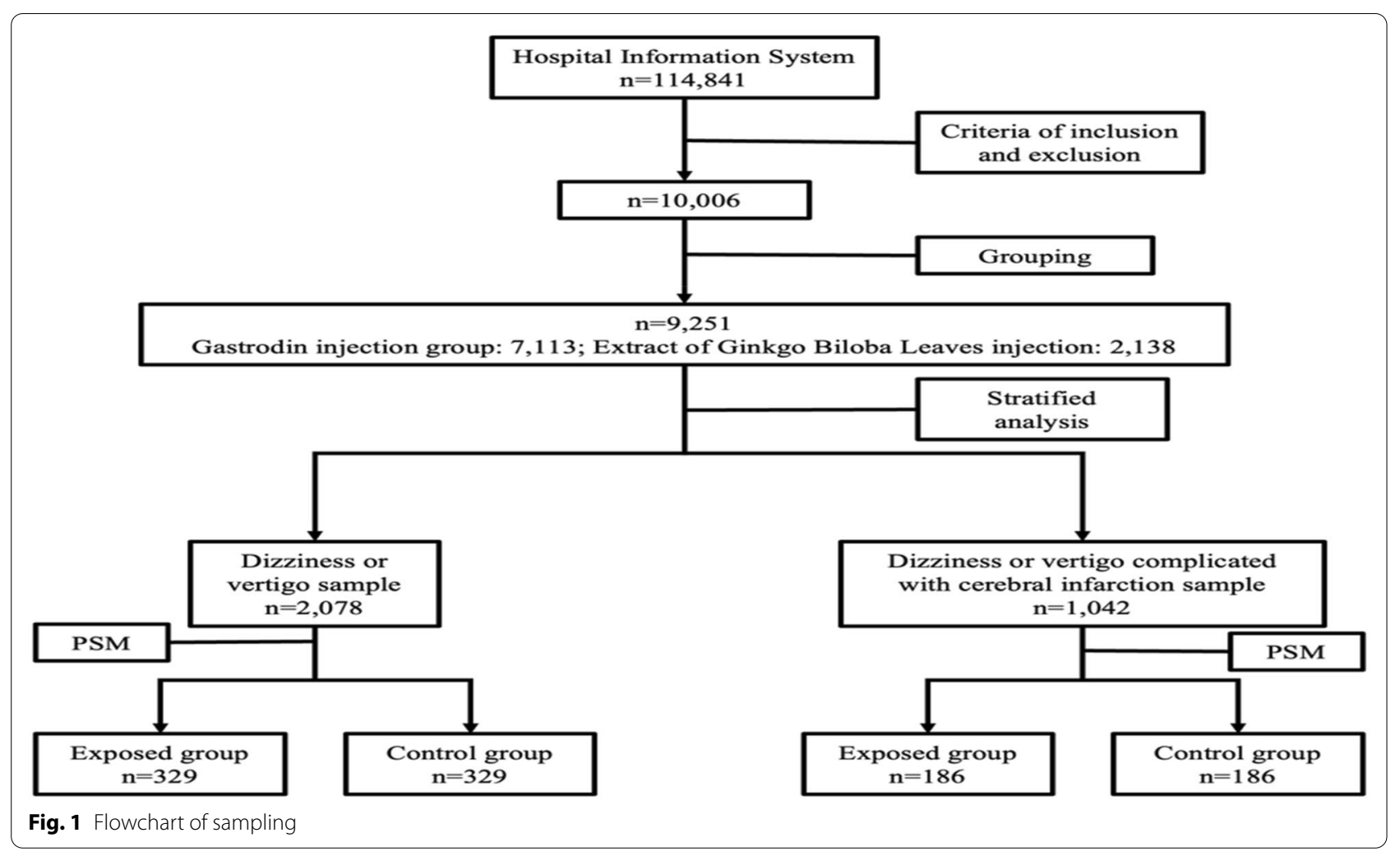

Table 1 Characteristics at baseline before PSM: sample of dizziness or vertigo

\begin{tabular}{|c|c|c|c|c|}
\hline Variable & Total $(\mathrm{N}=2057)$ & $\begin{array}{l}\text { Gastrodin injection } \\
(\mathrm{N}=1529)\end{array}$ & $\begin{array}{l}\text { Extract of Ginkgo Biloba Leaves } \\
\text { injection }(\mathrm{N}=528)\end{array}$ & $\mathbf{P}$ \\
\hline Region, (n, \%) & & & & $<0.001$ \\
\hline Eastern & $1079(52.5)$ & $824(53.9)$ & $255(48.3)$ & \\
\hline Central & $363(17.6)$ & $206(13.5)$ & $157(29.7)$ & \\
\hline Western & $556(27.0)$ & $449(29.4)$ & $107(20.3)$ & \\
\hline Northeast & $59(2.9)$ & $50(3.3)$ & $9(1.7)$ & \\
\hline Hospital level, (n, \%) & & & & $<0.001$ \\
\hline Tertiary & $1889(91.8)$ & $1384(90.5)$ & 505 (95.6) & \\
\hline Secondary & $139(6.8)$ & $122(8.0)$ & $17(3.2)$ & \\
\hline Primary & $29(1.4)$ & $23(1.5)$ & $6(1.1)$ & \\
\hline Hospital department, (n, \%) & & & & $<0.001$ \\
\hline Neurology & $1492(72.5)$ & $1179(77.1)$ & $313(59.3)$ & \\
\hline E.N.T & $146(7.1)$ & $37(2.4)$ & 109 (20.6) & \\
\hline Cardiovascular medicine & $29(1.4)$ & $23(1.5)$ & $6(1.1)$ & \\
\hline ICU & $390(19.0)$ & 0 & 0 & \\
\hline Others & $55(45,64)$ & $290(19.0)$ & $100(18.9)$ & \\
\hline Age (y), (n, \%) & & & & 0.674 \\
\hline$[18,45]$ & $514(25.0)$ & $377(24.7)$ & $137(25.9)$ & \\
\hline$[45,60]$ & $750(36.5)$ & $555(36.3)$ & $195(36.9)$ & \\
\hline$[60,75]$ & $758(36.8)$ & $568(37.1)$ & $190(36.0)$ & \\
\hline$\geq 75$ & $35(1.7)$ & $29(1.9)$ & $6(1.1)$ & \\
\hline Gender (n, \%) & & & & 0.959 \\
\hline Female & $1235(60.0)$ & $917(60.0)$ & $318(60.2)$ & \\
\hline Male & $822(40.0)$ & $612(40.0)$ & 210 (39.8) & \\
\hline
\end{tabular}




\section{Sample characteristics at baseline after PSM}

After PSM, the baseline was shown in Table 2. Gastrodin injection treatment group and Extract of Ginkgo Biloba Leaves injection treatment group finally included 329 patients, respectively. There was no difference at baseline between these two groups. In these two groups, it is mainly female, and the age is mainly 45 to 75 years old. There were about $60 \%$ patients come from eastern, about $97 \%$ patients come from tertiary hospitals and about $84 \%$ of them were treated in the Department of Neurology.

\section{Comparison of outcomes}

As shown in Table 3, compared with Extract of Extract of Ginkgo Biloba Leaves injection treatment, Gastrodin injection treatment had less hospitalization duration $(7(5,10)$ versus $8(6,10))$, but there was no any statistical significance $(P=0.080)$. There was a statistically significant difference between these two groups $(P<0.001)$ in terms of treatment effect. Specifically, the effective of Gastrodin injection treatment (100\%) was higher than Extract of Ginkgo Biloba Leaves injection treatment $(99.7 \%)$ with no significant difference $(P=1.000)$. However, the cure rate of Gastrodin injection treatment (42.9\%) was higher than Extract of Ginkgo Biloba Leaves injection treatment $(25.5 \%)$ with a significant difference $(P<0.05)$.

\section{Comparision of costs}

As shown in Table 4, the total hospitalization cost of Gastrodin injection treatment (5530.00 (4054.00, 7702.00)) was significantly $(P<0.001)$ less than that of Extract of Ginkgo Biloba Leaves injection treatment (6672.00 (5057.00, 9023.00)). Except for the other drug fee and treatment fee, the sub cost items of Gastrodin injection treatment were significantly $(P<0.001)$ less than that of Extract of Ginkgo Biloba Leaves injection treatment. Besides, Gastrodin injection treatment's nursing fee was less than Extract of Ginkgo Biloba Leaves injection treatment, but without significant difference $(P=0.056)$.

\section{Sample of dizziness or vertigo, with the complication of cerebral infarction \\ Sample characteristics at baseline before PSM}

Before PSM, 727 patients were included in Gastrodin injection treatment, while 315 patients included Extract of Ginkgo Biloba Leaves injection treatment. There were significant differences in region, hospital level, and age (see Table 5).

Table 2 Characteristics at baseline after PSM: sample of dizziness or vertigo

\begin{tabular}{|c|c|c|c|c|}
\hline Variable & $\begin{array}{l}\text { Gastrodin injection } \\
(\mathrm{N}=329)\end{array}$ & $\begin{array}{l}\text { Extract of Ginkgo Biloba Leaves } \\
\text { injection }(\mathrm{N}=329)\end{array}$ & $P$ & SMD \\
\hline Region, (n, \%) & & & 0.79 & 0.080 \\
\hline Eastern & $193(58.7)$ & $195(59.3)$ & & \\
\hline Central & $55(16.7)$ & $56(17.0)$ & & \\
\hline Western & $80(24.3)$ & $78(23.7)$ & & \\
\hline Northeast & $1(0.3)$ & $0(0.0)$ & & \\
\hline Hospital level, $(n, \%)$ & & & 1 & $<0.001$ \\
\hline Tertiary & $320(97.3)$ & $320(97.3)$ & & \\
\hline Secondary & $7(2.1)$ & $7(2.1)$ & & \\
\hline Primary & $2(0.6)$ & $2(0.6)$ & & \\
\hline Hospital department, (n, \%) & & & 0.909 & 0.058 \\
\hline Neurology & $279(84.8)$ & $276(83.9)$ & & \\
\hline E.N.T & $7(2.1)$ & $10(3.0)$ & & \\
\hline Cardiovascular medicine & $1(0.3)$ & $1(0.3)$ & & \\
\hline ICU & 0 & 0 & & \\
\hline Others & $42(12.8)$ & $42(12.8)$ & & \\
\hline Age $(y),(n, \%)$ & & & 0.991 & 0.026 \\
\hline$[18,45]$ & $63(19.1)$ & $60(18.2)$ & & \\
\hline$[45,60]$ & $133(40.4)$ & $133(40.4)$ & & \\
\hline$[60,75]$ & $128(38.9)$ & $131(39.8)$ & & \\
\hline$\geq 75$ & $5(1.5)$ & $5(1.5)$ & & \\
\hline Gender (n, \%) & & & 0.871 & 0.019 \\
\hline Female & $211(64.1)$ & $208(63.2)$ & & \\
\hline Male & $118(35.9)$ & $121(36.8)$ & & \\
\hline
\end{tabular}


Table 3 Outcome comparison after PSM: sample of dizziness or vertigo

\begin{tabular}{|c|c|c|c|}
\hline Variable & Gastrodin injection $(\mathrm{N}=329)$ & $\begin{array}{l}\text { Extract of Ginkgo Biloba Leaves injection } \\
(\mathrm{N}=329)\end{array}$ & $P$ \\
\hline Hospitalization duration, $M\left(Q_{L^{\prime}} Q_{U}\right)$ & $7(5,10)$ & $8(6,10)$ & 0.080 \\
\hline Hospitalization duration, (n, \%) & & & 0.022 \\
\hline 0 & $0(0.0)$ & $3(0.9)$ & \\
\hline$[1,7]$ & $169(51.4)$ & $139(42.2)$ & \\
\hline$[8,30]$ & $159(48.3)$ & $185(56.2)$ & \\
\hline$\geq 30$ & $1(0.3)$ & $2(0.6)$ & \\
\hline Treatment effect, $(n, \%)$ & & & $<0.001$ \\
\hline Death & 0 & 0 & \\
\hline Ineffective & 0 & 0 & \\
\hline Effective & $329(100)$ & $328(99.7)$ & 1.000 \\
\hline Turn for the better & $188(57.1)$ & $244(74.2)$ & \\
\hline Cure & $141(42.9)$ & $84(25.5)$ & $<0.05$ \\
\hline Others & $0(0.0)$ & $1(0.3)$ & \\
\hline
\end{tabular}

Table 4 Cost comparison after PSM: sample of dizziness or vertigo

\begin{tabular}{lllr}
\hline $\mathbf{M}\left(\mathbf{Q}_{\mathbf{L}}, \mathbf{Q}_{\mathbf{U}}\right)$ & Gastrodin injection $(\mathbf{N}=\mathbf{3 2 9})$ & $\begin{array}{l}\text { Extract of Ginkgo Biloba Leaves } \\
\text { injection }(\mathbf{N}=\mathbf{3 2 9})\end{array}$ & $\boldsymbol{P}$ \\
\hline Total hospitalization cost & & $6672.00(5057.00,9023.00)$ & $<0.001$ \\
Drug fees & $5530.00(4054.00,7702.00)$ & $1858.00(1121.00,2710.00)$ & $<0.001$ \\
$\quad$ Target drug fee & $1426.00(808.00,2446.00)$ & $746.10(483.80,1040.17)$ & $<0.001$ \\
$\quad$ Other drug fee & $326.16(206.52,464.67)$ & $1032.40(440.60,1758.44)$ & 0.169 \\
Inspection fee & $1087.73(507.11,2059.70)$ & $2056.00(1289.00,2745.00)$ & $<0.001$ \\
Laboratory fee & $1532.00(824.00,2078.00)$ & $1203.00(781.00,1533.00)$ & 0.001 \\
Treatment fee & $950.00(599.00,1454.00)$ & $348.00(220.00,752.00)$ & $<0.001$ \\
Bed fee & $573.00(244.00,1191.00)$ & $364.00(240.00,550.00)$ & 0.002 \\
Nursing fee & $300.00(200.00,480.00)$ & $164.00(108.00,255.00)$ & 0.056 \\
Operation fee & $146.00(99.00,220.00)$ & $0.00(0.00,0.00)$ & $<0.001$ \\
Other fee & $0.00(0.00,0.00)$ & $63.00(6.00,515.00)$ & 0.001 \\
Daily average target drug fee & $45.00(1.00,123.00)$ & $96.76(75.26,120.95)$ & $<0.001$ \\
Daily average hospitalization fee & $51.63(34.42,58.08)$ & $842.83(645.90,1102.71)$ & 0.029 \\
Per capita hospitalization cost, mean $\pm S D$ & $784.33(544.30,1089.33)$ & $7165.25 \pm 3275.85$ & $<0.001$ \\
\hline
\end{tabular}

\section{Sample characteristics at baseline after PSM}

After PSM, the baseline was shown in Table 6. Gastrodin injection treatment group and Extract of Ginkgo Biloba Leaves injection treatment group finally included 186 patients, respectively. There was no difference at baseline between these two groups. In these two groups, it is mainly female (52.2\%), and the age is mainly 60 to 75 years old. There were $48.4 \%$ patients come from eastern and $38.7 \%$ central, $99.5 \%$ patients come from tertiary hospitals and about $81.2 \%$ of them were treated in the Department of Neurology.

\section{Comparison of outcomes}

As shown in Table 7, between Gastrodin injection treatment and Extract of Ginkgo Biloba Leaves injection treatment, there was no significant difference for hospitalization duration $(P=0.371)$, but a significant difference for the treatment effect $(P=0.009)$. Specifically, the effective of Gastrodin injection treatment (99.0\%) was less than Extract of Ginkgo Biloba Leaves injection treatment $(100 \%)$ with no significant difference $(P=0.499)$. However, the cure rate of Gastrodin injection treatment (32.3\%) was higher than Extract of Ginkgo Biloba Leaves 
Table 5 Characteristics at baseline before PSM: sample of dizziness or vertigo, with the complication of cerebral infarction

\begin{tabular}{|c|c|c|c|c|}
\hline Variable & Total $(\mathrm{N}=1042)$ & $\begin{array}{l}\text { Gastrodin injection } \\
(\mathrm{N}=727)\end{array}$ & $\begin{array}{l}\text { Extract of Ginkgo Biloba Leaves } \\
\text { injection }(\mathrm{N}=315)\end{array}$ & $P$ \\
\hline Region, $(\mathrm{n}, \%)$ & & & & $<0.001$ \\
\hline Eastern & $427(41.0)$ & $323(44.4)$ & $104(33.0)$ & \\
\hline Central & $305(29.3)$ & $136(18.7)$ & $169(53.7)$ & \\
\hline Western & $228(21.9)$ & $205(28.2)$ & $23(7.3)$ & \\
\hline Northeast & $82(7.9)$ & $63(8.7)$ & $19(6.0)$ & \\
\hline Hospital level, (n, \%) & & & & 0.001 \\
\hline Tertiary & $983(94.3)$ & $677(93.1)$ & $306(97.1)$ & \\
\hline Secondary & $32(3.1)$ & $31(4.3)$ & $1(0.3)$ & \\
\hline Primary & $27(2.6)$ & $19(2.6)$ & $8(2.5)$ & \\
\hline Hospital department, (n, \%) & & & & 0.064 \\
\hline Neurology & $776(74.5)$ & $541(74.4)$ & $235(74.6)$ & \\
\hline E.N.T & $18(1.7)$ & 0 & 0 & \\
\hline Cardiovascular medicine & $1(0.1)$ & $17(2.3)$ & $1(0.3)$ & \\
\hline $\mathrm{ICU}$ & $247(23.7)$ & $1(0.1)$ & $0(0.0)$ & \\
\hline Others & $64(56,69)$ & $168(23.1)$ & $79(25.1)$ & \\
\hline Age $(y),(n, \%)$ & & & & 0.022 \\
\hline$[18,45]$ & $35(3.4)$ & $19(2.6)$ & $16(5.1)$ & \\
\hline$[45,60]$ & $314(30.1)$ & $230(31.6)$ & $84(26.7)$ & \\
\hline$[60,75]$ & $657(63.1)$ & $448(61.6)$ & $209(66.3)$ & \\
\hline$\geq 75$ & $36(3.5)$ & $30(4.1)$ & $6(1.9)$ & \\
\hline Gender $(n, \%)$ & & & & 0.281 \\
\hline Female & $510(48.9)$ & $364(50.1)$ & $146(46.3)$ & \\
\hline Male & $532(51.1)$ & 363 (49.9) & 169 (53.7) & \\
\hline
\end{tabular}

injection treatment $(21.0 \%)$ with a significant difference $(P<0.05)$.

\section{Comparision of costs}

As shown in Table 8, the total hospitalization cost of Gastrodin injection treatment $(7391.47$ (5030.42, 10,654.81)) was less than that of Extract of Ginkgo Biloba Leaves injection treatment $(8212.60(5243.71,12,633.69))$ with no significant difference $(P=0.123)$. In terms of the drug fee, target drug fee, inspection fee, daily average target drug fee, and per capita hospitalization cost, Gastrodin injection group was significantly $(P<0.001)$ less than that of Extract of Ginkgo Biloba Leaves injection treatment.

\section{Discussion}

In this study, we focused on the clinical and economic anlaysis of Gastrodin injection, comparing with Extract of Ginkgo Biloba Leaves injection, which is commonly used to treat dizziness or vertigo [50, 51]. We compared the Extract of Ginkgo Biloba Leaves injection with Gastrodin injection through clinical and economic evaluation outcome indicators such as medical cost of hospitalization, days of hospital stay, effective rate, and cure rate, to analyze the drug economy and effectiveness of Gastrodin injection in the real world. The data of this study was from real-world, not from controlled clinical trials. Real-world data is different from controlled clinical trial data, real-world evidence provides external validity and can provide evidence for the effectiveness and economic effects [52]. Real-world evidence can be used to complement data from controlled clinical trials to enable the generalization of clinical findings to a more inclusive and larger population, it can provide instructive information for clinical practice [53]. The correlations of gastrodin injection in the treatment of dizziness or vertigo were analyzed, and the differences of these two groups were found. So, this study's results can provide valuable reference for the clinical practice of dizziness or vertigo.

Nevertheless, the use of real-world data is prone to pose selection bias challenges [54]. Therefore, to reduce the impact of confounding factors on intervention effect estimation, stratified analysis and PSM were used in this study. After PSM, the baseline situation between the exposed and control groups was more balanced and comparable than before, and some significant findings were generated that are worth further discussion below.

Regarding hospitalization duration, it found that there were no significant differences that of these two study 
Table 6 Characteristics at baseline after PSM: sample of dizziness or vertigo, with the complication of cerebral infarction

\begin{tabular}{|c|c|c|c|c|}
\hline Variable & $\begin{array}{l}\text { Gastrodin injection } \\
(\mathrm{N}=186)\end{array}$ & $\begin{array}{l}\text { Extract of Ginkgo Biloba Leaves } \\
\text { injection }(N=186)\end{array}$ & $P$ & SMD \\
\hline Region, (n, \%) & & & 1 & $<0.001$ \\
\hline Eastern & $90(48.4)$ & $90(48.4)$ & & \\
\hline Central & $72(38.7)$ & $72(38.7)$ & & \\
\hline Western & $15(8.1)$ & $15(8.1)$ & & \\
\hline Northeast & $9(4.8)$ & $9(4.8)$ & & \\
\hline Hospital level, (n, \%) & & & 1 & $<0.001$ \\
\hline Tertiary & $185(99.5)$ & $185(99.5)$ & & \\
\hline Secondary & 0 & 0 & & \\
\hline Primary & $1(0.5)$ & $1(0.5)$ & & \\
\hline Hospital department, (n, \%) & & & 1 & $<0.001$ \\
\hline Neurology & $151(81.2)$ & $151(81.2)$ & & \\
\hline E.N.T & 0 & 0 & & \\
\hline Cardiovascular medicine & 0 & 0 & & \\
\hline ICU & 0 & 0 & & \\
\hline Others & $35(18.8)$ & $35(18.8)$ & & \\
\hline Age (y), (n, \%) & & & 1 & $<0.001$ \\
\hline$[18,45]$ & $4(2.2)$ & $4(2.2)$ & & \\
\hline$[45,60]$ & $55(29.6)$ & 55 (29.6) & & \\
\hline$[60,75]$ & $123(66.1)$ & $123(66.1)$ & & \\
\hline$\geq 75$ & $4(2.2)$ & $4(2.2)$ & & \\
\hline Gender (n, \%) & & & 1 & $<0.001$ \\
\hline Female & $97(52.2)$ & $97(52.2)$ & & \\
\hline Male & $89(47.8)$ & 89 (47.8) & & \\
\hline
\end{tabular}

Table 7 Outcome comparison after PSM: sample of dizziness or vertigo, with the complication of cerebral infarction

\begin{tabular}{|c|c|c|c|}
\hline Variable & Gastrodin injection $(\mathrm{N}=186)$ & $\begin{array}{l}\text { Extract of Ginkgo Biloba Leaves injection } \\
(\mathrm{N}=186)\end{array}$ & $P$ \\
\hline Hospitalization duration, $M\left(\mathrm{Q}_{L^{\prime}} \mathrm{Q}_{U}\right)$ & $9(7,12)$ & $10(7,13)$ & 0.371 \\
\hline Hospitalization duration, (n, \%) & & & 0.516 \\
\hline 0 & $2(1.1)$ & $0(0.0)$ & \\
\hline$[1,7]$ & $57(30.6)$ & $55(29.6)$ & \\
\hline$[8,30]$ & $127(68.3)$ & $130(69.9)$ & \\
\hline$\geq 30$ & $0(0.0)$ & $1(0.5)$ & \\
\hline Treatment effect, $(n, \%)$ & & & 0.009 \\
\hline Death & 0 & 0 & \\
\hline Ineffective & 0 & 0 & \\
\hline Effective & $184(99.0)$ & $186(100)$ & 0.499 \\
\hline Turn for the better & $124(66.7)$ & $147(79.0)$ & \\
\hline Cure & $60(32.3)$ & $39(21.0)$ & $<0.05$ \\
\hline Others & $2(1.1)$ & $0(0.0)$ & \\
\hline
\end{tabular}

groups in the sample of dizziness or vertigo and the sample of dizziness or vertigo complicated with cerebral infarction. In the past clinical study, it found that the combination of Gastrodin injection and conventional treatment had significant $(P<0.05)$ advantages in the improvement time compared with conventional treatment for the treatment of vertigo [55].

Regarding medical cost per hospitalization, this study found that the medical const per hospitalization of Gastrodin injection group was significantly $(P<0.001)$ lower 
Table 8 Cost comparison after PSM: sample of dizziness or vertigo, with the complication of cerebral infarction

\begin{tabular}{llll}
\hline $\mathbf{M}\left(\mathbf{Q}_{\mathbf{L}}, \mathbf{Q}_{\mathbf{U}}\right)$ & Gastrodin injection $(\mathbf{N}=\mathbf{1 8 6})$ & $\begin{array}{l}\text { Extract of Ginkgo Biloba Leaves } \\
\text { injection }(\mathbf{N}=\mathbf{1 8 6})\end{array}$ & $\boldsymbol{P}$ \\
\hline Total hospitalization cost & $7391.47(5030.42,10,654.81)$ & $8212.60(5243.71,12,633.69)$ & 0.123 \\
Drug fees & $2671.15(1501.84,4144.48)$ & $2883.27(1881.75,4982.69)$ & 0.039 \\
$\quad$ Target drug fee & $361.41(206.52,516.30)$ & $677.32(387.04,1064.36)$ & $<0.001$ \\
$\quad$ Other drug fee & $2240.97(1152.53,3842.02)$ & $2101.26(1254.55,4199.22)$ & 0.661 \\
Inspection fee & $1635.00(1054.25,2206.66)$ & $1926.00(1124.50,2880.00)$ & 0.005 \\
Laboratory fee & $1209.50(722.15,1776.38)$ & $1079.00(825.35,1910.65)$ & 0.687 \\
Treatment fee & $566.33(235.75,1272.78)$ & $403.00(172.37,874.44)$ & 0.147 \\
Bed fee & $410.00(250.00,560.00)$ & $420.00(251.03,599.88)$ & 0.692 \\
Nursing fee & $222.19(126.47,347.91)$ & $206.76(141.69,381.78)$ & $0.00(0.00,0.00)$ \\
Operation fee & $0.00(0.00,0.00)$ & $75.60(5.20,312.00)$ & 0.712 \\
Other fee & $87.50(20.50,325.12)$ & $85.83(42.63,106.22)$ & 0.761 \\
Daily average target drug fee & $51.63(25.82,57.37)$ & $872.35(670.06,1212.33)$ & 0.293 \\
Daily average hospitalization fee & $824.75(609.41,1120.93)$ & $11,715.70 \pm 14,086.39$ & $<0.001$ \\
Per capita hospitalization cost, mean \pm SD & $9315.75 \pm 9472.04$ & 0.122 \\
\hline
\end{tabular}

than of Extract of Ginkgo Biloba Leaves injection group in the sample of dizziness or vertigo, but no significant differences that of these two study groups in the sample of dizziness or vertigo complicated with cerebral infarction. The economic advantage of Gastrodin injection for dizziness or vertigo patients can be proven in this study.

Although Gastrodin injection is effective in treating dizziness or vertigo [56], it also has some adverse drug reactions (ADRs) occasionally, such as dry mouth and nose, local pain, rash, and somnolence, etc. Based on two meta-analyses, few studies reported ADRs, while reported ADRs were mild and did not need special treatment $[27,28]$. In a multi-center single-blind randomized controlled trial, of the 120 patients in the Gastrodin injection group, 10 had adverse reactions; the ADRs rate was $8.33 \%$ in the Gastrodin injection group and $10.83 \%$ in the control group without statistical significance $(P=0.538)$ [29]. However, most of the ADRs will return to normal after stopping the drug. Therefore, although the ADRs rate reported in the past studies is low and mild, Gastrodin injection safety still needs to be further clinical verification. It implies that patient-centered clinical medication needs more exploration.

Also, the strengths of this study are obvious. This study reflects the correlation of gastrodin injection in the treatment of dizziness or vertigo, and also shows that there are differences between Gastrodin injection and Extract of Ginkgo Biloba Leaves injection. Firstly, the actual clinical practice can be shown by using real-world data to conduct the clinical and economic evaluation of Gastrodin injection for dizziness or vertigo. Secondly, the data of this study were collected from 131 hospitals across the country, which covered the whole nation, so the findings have more generalizing significance for dizziness or vertigo treatment. Thirdly, there is still lacking studies to evaluate the clinical and economic benefit of the Gastrodin injection with the comparison of other drugs for patients in the actual clinical practice. Therefore, this study supplemented the evidence in this respect.

\section{Limitations and future research}

However, this study has some limitations which can be addressed in future studies. First, this study recorded the realistic drug utilization data without the standardized dosage of different treatments in the real-world data of electronic health record data. Prospective desing of pragmatic controlled trial with a standardized therapeutic dosage in the future studies can generate more direct evidence of Gastrodin injection on dizziness and vertigo. Second, it is impossible to eliminate the influence of all confounding factors, such as Charlson Comorbidity Index (CCI), disease course, concomitant medication, cerebral infarction subclass, etc. Future studies can particularly explore the effects of Gastrodin Injection on dizziness and vertigo in specific disease areas. Third, this research only compared two naturally extracted medicines without considering conventional medicine. Thus, future studies can use conventional medicines as comparators to test the two naturally extracted medicines' clinical effects. Fourth, hospital policy, insurance policies, and pharmaceutical factories can influence drug utilization. However, in this research, we only focus on the personal patient level by using individual data of electronic medical records to test the clinical and economic effects of two naturally extracted medicines. The impacts of these 
contextual factors need further exploration in future studies. Last, the sample patients of this study only from inpatients, the findings can provide references for clinicians to optimize treatment options and for health insurance departments to make relevant policies. But may not reflect the whole disease populations without outpatients. Thus, future studies can include outpatients and inpatients for the comprehensive research.

\section{Conclusions}

Gastrodin injection showed advantages for inpatients with dizziness or vertigo compared with Extract of Ginkgo Biloba Leaves injection. Future studies using prospective pragmatic controlled trials can test and explore more about the effects of Gastrodin injections on dizziness or vertigo.

\section{Abbreviations}

ADRs: Adverse drug reactions; HIS: Hospital Information System; PSM: Propensity score matching; 4-HBAL: 4-Hydroxybenzaldehyde; 4-HBA: 4-Hydroxybenzyl alcohol; PPS: Per Protocol Set; NNM: Nearest Neighbor Matching; CCl: Charlson Comorbidity Index.

\section{Supplementary Information}

The online version contains supplementary material available at https://doi. org/10.1186/s13020-021-00561-9.

Additional file 1: STROBE Statement-Checklist of items that should be included in reports of cohort studies.

\section{Acknowledgements}

The authors gratefully acknowledge the support from the doctors who gave professional advice on this study.

\section{Authors' contributions}

YL, RW, SH and HH conceptualized and designed the study. All the authors collected data, conducted data analysis and data interpretation. YL, RW, WL, $\mathrm{NL}$, and $\mathrm{HH}$ drafted the manuscript. COLU participated into manuscript revision. All authors have read and approved the final manuscript.

\section{Funding}

This research was supported by a grant from the National Natural Science Foundation of China ("Component Analysis of Increased Drug Expenditure in China Hospitals Based on Non-Stochastic Index-Theoretical Model: $\left.71603008^{\prime \prime}\right)$.

\section{Availability of data and materials}

The data can be provided from corresponding authors upon reasonable request.

\section{Declarations}

Ethics approval and consent to participate

The studies were reviewed and approved by the Ethics Committee of Peking University (71603008).

\section{Consent for publication}

Consent for publication was waived by the ethic committee because this is a retrospective study which only included anonymous dataset.

\section{Competing interests}

The authors declare that the research was conducted in the absence of any commercial or financial relationships that could be construed as a potential conflict of interes.

\section{Author details}

${ }^{1}$ School of Public Health and Management, Guangzhou University of Chinese Medicine, Guangzhou, China. ${ }^{2}$ State Key Laboratory of Quality Research in Chinese Medicine, Institute of Chinese Medical Sciences, University of Macau, Taipa, Macao SAR, China. ${ }^{3}$ Department of Continuing Medical Education, Peking University Health Science Center, Beijing, China. ${ }^{4}$ Department of Real-World Evidence and Pharmacoeconomics, International Research Center for Medicinal Administration, Peking University, Beijing, China. ${ }^{5}$ Department of Vasculocardiology, AnZhen Hospital, Affiliated to Capital Medical University, Beijing, China. ${ }^{6}$ Inchuan Medlinker Internet Hospital, Yinchuan, NingXia Hui Autonomous Region, China.

Received: 14 April 2021 Accepted: 25 December 2021

Published online: 04 January 2022

References

1. Drachman DA, Hart CW. An approach to the dizzy patient. Neurology. 1972;22:323-34.

2. Post RE, Dickerson LM. Dizziness: a diagnostic approach. Am Fam Physician. 2010;82(4):361-8.

3. Qi XK, Wang XF. Master the concept, classification and diagnostic process of dizziness. Transl Med J. 2016;5(1):1-4. https://doi.org/10.3969/j.issn. 2095-3097.2016.01.001 (in Chinese)

4. Jahn K, Kressig RW, Bridenbaugh SA, Brandt T, Schniepp R. Dizziness and unstable gait in old age: etiology, diagnosis and treatment. Dtsch Arztebl Int. 2015;112(23):387-93. https://doi.org/10.3238/arztebl.2015.0387.

5. Lu LT, Wang HW, Wang HM, Wang JD, Sang JZ. Clinical observation of salvianolate for injection in treatment of acute cerebral infarction with dizziness/vertigo as first symptom. Drug Eval Res. 2020;43(3):506-8. https://doi.org/10.7501/j.issn.1674-6376.2020.03.026 (in Chinese).

6. Zhu CT, Ju Y. Cerebrovascular disease and vertigo or dizziness. Chin J Stroke. 2018;13(3):284-8. https://doi.org/10.3969/j.issn.1673-5765.2018. 03.019 (in Chinese)

7. Chen YH, Wang Y, Xu L, Li W, Luo R, Gong T, et al. Clinical manifestations and risk factors in posterior circulation ischemic stroke. Chin J Cardiovasc Med. 2014;19(2):101-4. https://doi.org/10.3969/j.issn.1007-5410.2014.02. 006 (in Chinese).

8. Kerber KA, Meurer WJ, Brown DL, Burke JF, Hofer TP, Tsodikov A, et al. Stroke risk stratification in acute dizziness presentation: a prospective imaging-based study. Neurology. 2015;85(21):1869-78. https://doi.org/ 10.1212/WNL.0000000000002141.

9. Bai HX, Xiao Y, Zhao X, Wang ZL, Kasner SE, Yang L, et al. Early identification of cerebrovascular events in patients presenting with vertigo or dizziness. Int J Stroke. 2016;1 1(6):64-7. https://doi.org/10.1177/1747493016 632250.

10. GBD 2013 DALYs and HALE Collaborators, Murray CJ, Barber RM, Foreman KJ, Ozgoren AA, Abd-Allah F, et al. Global, regional, and national disabilityadjusted life years (DALYs) for 306 diseases and injuries and healthy life expectancy (HALE) for 188 countries, 1990-2013: quantifying the epidemiological transition. Lancet. 2015;386(10009):2145-91. https://doi. org/10.1016/S0140-6736(15)61340-X.

11. Wang WZ, Jiang B, Sun HX, Ru XJ, Sun DL, Wang LH, et al. Prevalence, incidence, and mortality of stroke in China: results from a nationwide population-based survey of 480687 adults. Circulation. 2017;135(8):75971. https://doi.org/10.1161/CIRCULATIONAHA.116.025250.

12. Bejot $Y$, Delpont $B$, Giroud M. Rising stroke incidence in young adults: more epidemiological evidence, more questions to be answered. J Am Heart Assoc. 2016;5: e003661. https://doi.org/10.1161/JAHA.116.003661.

13. Gulli G, Marquardt L, Rothwell PM, Markus HS. Stroke risk after posterior circulation stroke/transient ischemic attack and its relationship to site of vertebrobasilar stenosis: pooled data analysis from prospective studies. Stroke. 2013;44(3):598-604. https://doi.org/10.1161/STROKEAHA.112. 669929. 
14. LiTM, Mao JL, Zhong P, Wu Y, Chen B. Clinical application of self-rating somatization symptoms scale in silent brain infarction patients with complaints of dizziness. Stroke Nerv Dis. 2018;25(2):159-62. https://doi.org/10.3969/jissn. 1007-0478.2018.02.010 (in Chinese).

15. Chinese Society of Neurology, Chinese Medical Association, Editorial Board of Chinese Journal of Neurology. Multidisciplinary expert consensus of diagnosis and treatment of vertigo. Chin J Neurol. 2017;50(11):805-12. https:/doi.org/ 10.3760/cma.jissn. 1006-7876.2017.11.002 (in Chinese).

16. Wang C, Yu B, Kong WJ. Research progresses of the pharmacology of Gastrodia elata Bl. and gastrodin in improving glucose and lipid metabolism disorders. China Med Her. 2016;13(27):51-8 (in Chinese).

17. Yu B, Zuo ZY, Kong WJ. Experimental studies of the toxicity and safety of Gastrodina elata powder tablets. China Mod Med. 2014;21 (21):6-10 (in Chinese)

18. Liu Y, Gao JL, Peng M, Meng HY, Ma HB, Cai PP, et al. A review on central nervous system effects of Gastrodin. Front Pharmacol. 2018;9(24):1-18. https://doi. org/10.3389/fphar.2018.00024.

19. Zhou HY, Wu L, Ding LQ. Evaluation of efficacy and safety of gastrodine in treatment of elder patients with dizziness. Chin J Pharmacol Ther. 2003;8(4):471-2 (in Chinese).

20. Zhao XM, Zou Y, Xu H, Fan L, Gau HY, Li XM, et al. Gastrodin protect primary cultured rat hippocampal neurons against amyloid-beta peptide-induced neurotoxicity via ERK1/2-Nrf2 pathway. Brain Res. 2012;1482:13-21. https://doi. org/10.1016/j.brainres.2012.09.010.

21. Yong W, Xing TR, Wang S, Chen L, Hu P, Li CC, et al. Protective effects of gastrodin on lead-induced synaptic plasticity deficits in rat hippocampus. Planta Med. 2009;75(10):1112-7. https://doi.org/10.1055/s-0029-1185452.

22. Zhang FY, Hong SJ, Zhao LP, Li LH. Research advances of protective effect and mechanism of Gastrodin on nervous system. J Henan Univ Sci Tech (Med Sci). 2019;37(3):236-40. https://doi.org/10.15926/j.cnki.issn1672-688x.2019.03.022 (in Chinese).

23. Kim IS, Choi DK, Jung HJ. Neuroprotective effects of vanillyl alcohol in Gastrodia elata Blume through suppression of oxidative stress and antiapoptotic activity in toxin-induced dopaminergic MN9D cells. Molecules. 2011;16(7):5349-61. https://doi.org/10.3390/molecules16075349.

24. Kim BW, Koppula S, Kim JW, Lim HW, Hwang JW, Kim IS, et al. Modulation of LPS-stimulated neuroinflammation in BV-2 microglia by Gastrodia elata: 4-Hydroxybenzyl alcohol is the bioactive candidate. J Ethnopharmacol. 2012;139(2):549-57. https://doi.org/10.1016/j.jep.2011.11.048.

25. Ni HJ, Qin SG, Wang YL. Clinical application of Tianma injection. Clin J Chin Med. 2012;4(1):100-1 (in Chinese)

26. Li S, Xia H, Liu ZB, Liang GC. Advances in research of gastrodin in cardiovascular and cerebrovascular diseases. Chin Med J Res Prac. 2019;33(1):76-81. https://doi.org/10.13728/j.1673-6427.2019.01.018.

27. Ye CX, Jiang SY, Fang J. Meta-analysis of gastrodin injection in the treatment of vertigo. China Modern Med. 2009;16(22):52-4 (in Chinese).

28. Zhou BH, Chen P, Luo Y. Efficacy and safely of Tianmasu injection in treatment of acute cerebral infarction: a meta analysis. China Med. 2018;13(11):1655-60. https://doi.org/10.3760/j.issn.1673-4777.2018.11.015 (in Chinese).

29. Chen J, Liu SX, Hu GH, Zhang XY, Xi Y. Gastrodin injection in the treatment of vertigo: a multi-center single-blind randomized controlled trial. Chin J Evid Based Med. 2004;4(12):864-70 (in Chinese).

30. Pan YY. Application effect of Gastrodin injection combined with diphenhydramine hydrochloride injection in patients with acute vertigo. China Mod Med. 2020:27(10):74-6 (in Chinese).

31. Li ZY. Analysis on clinical efficacy of Banxia Baizhu Tianma decoction combined with Gastrodin injection in the treatment of wind-phlegm disturbance type vertigo. Clin Med Eng. 2020;27(3):309-10. https://doi.org/ 10.3969/j.issn.1674-4659.2020.03.0309.

32. He WJ. Clinical effect of self-designed Xifeng Huatan decoction combined with Gastrodin injection in the treatment of vertigo. China Mod Med. 2019;26(32):169-71 (in Chinese).

33. Tian F, Xie YM. Real-world study: a potential new approach to effectiveness evaluation of traditional Chinese medicine interventions. J Chin Integr Med. 2010;8(4):301-6. https://doi.org/10.3736/jcim20100401 (in Chinese).

34. Liang Z, Lai YF, Li M, Shi JN, Lei CL, Hu H, Ung COL. Applying regulatory science in traditional Chinese medicines for improving public safety and facilitating innovation in China: a soping review and regulatory implications. Chin Med. 2021;16(23):1-16. https://doi.org/10.1186/s13020-021-00433-2.

35. Zhang PF, Liao $\sqcup$, Deng Z, Tan YP. Research progress of pharmacological effects and clinical application of Ginkgo biloba extract. Liaoning J Tradit
Chin Med. 2017;44(2):426-9. https://doi.org/10.13192/j.issn.1000-1719.2017. 02.069 .

36. Liu N, Yin LH, Zhao G. Effect of Ginkgo biloba extract combined with alprostadil on central vertigo and its effect on patients blood rheology. Shaanxi J Tradit Chin Med. 2020;41(7):868-70. https://doi.org/10.3969/i.ssn.1000-7369. 2020.07 .008 (in Chinese).

37. Wang YK, Luo YY, Lei J, Yang F, Wang C, Meng S. Clinical observation of extract of Ginkgo biloba leaves injection combined with flunarizine in treatment of Meniere's disease. Drug Clinic. 2019;34(3):627-30. https://doi.org/10.7501/j. issn. 1674-5515.2019.03.010

38. Fan YD, Wang Y, Yu S, Chang J, Yan YQ, Wang YY, Bian YH. Natural products provide a new perspective for anti-complement treatment of severe COVID-19: a review. Chin Med. 2021;16(67):1-15. https://doi.org/10.1186/ s13020-021-00478-3.

39. Li XZ, Chen WJ, Simal-Gandara J, Georgiev MI, Li HY, Hu H, Wu X, Efferth T, Wang SP. West meets east: open up a dialogue on phytomedicine. Chin Med. 2021;16(57):1-18. https://doi.org/10.1186/s13020-021-00467-6.

40. Yuan Y, Jin XH, Liu J, Zhao X, Zhou JH, Wang X, et al. The Gastrodia elata genome provides insights into plant adaptation to heterotrophy. Nat Commun. 2018;9:1615. https://doi.org/10.1038/s41467-018-03423-5.

41. Chow S, Chen SC. Notes on Chinese Gastrodia. Acta Bot Yunnanica. 1983;5(4):361-8 (in Chinese).

42. Li Y, Wang ZW, Liu DH, Wang X, Zhou HL. Research advances in chemical constituents of Gastrodia elata Blume. Shandong Sci. 2016;29(4):24-9. https://doi.org/10.3976/j.issn.1002-4026.2016.04.006.

43. Chinese Academy of Sciences. Flora of China. Beijing: Science Press; 2004. (in Chinese).

44. Liu XD, Zhang Y, Wu MH, Ma ZG, Huang ZH, Tian F, Dong SH, Luo SM, Zhou Y, Zhang JJ, Li NX, He XF, Cao H. The scientific elucidation of daodi medicinal materials. Chin Med. 2020;15(86):1-11. https://doi.org/10.1186/ s13020-020-00367-1.

45. Chinese Pharmacopoeia Commission. Pharmacopoeia of the People's Republic of China. Beijing: Chinese Medical Science and Technology Press; 2015. (in Chinese).

46. Jang JH, Son YH, Kang SS, Bae CS, Kim JC, Kim SH, et al. Neuropharmacological potential of Gastrodia elata Blume and its components. Evid Based Compl Alt. 2015;309261:14. https://doi.org/10.1155/2015/309261.

47. Zhou J, Yang YB, Yang JR. The chemistry of Gastrodia elata BL. Acta Chim Sinica. 1979;37(3):183-9 (in Chinese).

48. Feng XZ, Chen YW, Yang JS. Studies on constituents of Tian-ma. Acta Chim Sinica. 1979;37(3):175-82 (in Chinese).

49. Duan XH, Li ZL, Yang DS, Zhang FL, Lin Q, Dai R. Study on the chemical constituents of Gastrodia elata. J Chin Med Mater. 2013;36(10):1608-11. https:// doi.org/10.13863/j.issn1001-4454.2013.10.022 (in Chinese).

50. Zhou HJ. A review on pharmacological action of active principle in gastrodia elata and the clinical application. Clin J Chin Med. 2016;8(22):56-8 (in Chinese).

51. Yang Y, Zhou B, Zhao WJ. Ginkgo biloba leaves history: a model of research and development for Chinese materia medica/phytomedicine. Chin Tradit Herb Drugs. 2016;47(15):2579-91. https://doi.org/10.7501/j.issn.0253-2670. 2016.15.001

52. Bowrin K, Briere JB, Levy P, Toumi M, Millier A. Use of real-world evidence in meta-analyses and cost-effectiveness models. J Med Econ. 2020;23(10):1053-60. https://doi.org/10.1080/13696998.2020.1792917.

53. Mphil NJ, Espinoza MA, Ratto B, Nicholson M, Rosselli D, Ovcinnikova O. Real-world evidence in healthcare decision making: cgobal trends and case studies from Latin America. Value Health. 2019;22(6):739-49. https://doi.org/ 10.1080/13696998.2019.1588737.

54. Bowrin K, Briere JB, Levy P, Millier A, Clay E, Toumi M. Cost-effectiveness analysis using real-world data: an overview of the literature. J Med Econ. 2019;22(6):545-53. https://doi.org/10.1080/13696998.2019.1588737.

55. Liu DD, Yang W. Clinical effect of Gastrodin injection on vertigo and tinnitus. China Contin Med Educ. 2019;11(15):131-3. https://doi.org/10.3969/j.issn. 1674-9308.2019.14.056 (in Chinese).

56. Zhou Y. Clinical efficiency and safety of Gastrodin injection in the treatment of systematic vertigo. China Pharmacist. 2016;19(11):2094-6 (in Chinese).

\section{Publisher's Note}

Springer Nature remains neutral with regard to jurisdictional claims in published maps and institutional affiliations. 